\title{
Tregs Biomimetic Nanoparticle to Reprogram Inflammatory and Redox Microenvironment in Infarct Tissue to Inhibit the Remodeling of the Left Ventricle of Myocardial Ischemia Reperfusion Injury in Mice
}

\section{Fangyuan Li}

Air Force Medical University

Daozhou Liu

Air Force Medical University

Miao Liu

Air Force Medical University

Qifeng Ji

Air Force Medical University

\section{Bangle Zhang}

Air Force Medical University

Qibing Mei

Air Force Medical University

Ying Cheng

Air Force Medical University

Si-yuan Zhou ( $\square$ zhousy@fmmu.edu.cn )

Air Force Medical University https://orcid.org/0000-0001-6092-4406

\section{Research Article}

Keywords: myocardial ischemia reperfusion injury, regulatory T cells, platelet membrane, cyclosporin A, reactive oxygen species, macrophage

Posted Date: December 8th, 2021

DOI: https://doi.org/10.21203/rs.3.rs-1125947/v1

License: (c) (i) This work is licensed under a Creative Commons Attribution 4.0 International License.

Read Full License 


\section{Abstract \\ Background}

At present, patients with myocardial infarction remain an increased risk for myocardial ischemia/reperfusion injury (MI/RI), which currently lacks an effective therapeutic method. It is still a bottleneck that effectively deliver drug to ischemic myocardium to treat MI/RI. Inspired by the protective effect of regulatory $\mathrm{T}$ cells (Tregs) on MI/RI and natural role of platelets in adhesion with damaged blood vessel in heart during myocardial infarct, a Tregs biomimetic nanoparticle (CsA@PPTK) was prepared by camouflaging a cyclosporin A (CsA)-loaded and reactive oxygen species (ROS)-sensitive nanoparticle with platelet membrane.

\section{Results}

In MI/RI mice, CsA@PPTK could be preferentially delivered to ischemic myocardium. CsA@PPTK significantly scavenged ROS in ischemic myocardium, while it also markedly increased the generation of Tregs and the ratio of $\mathrm{M} 2$ type macrophage to $\mathrm{M} 1$ type macrophage in ischemic myocardium. Furthermore, CsA@PPTK significantly attenuated apoptosis of cardiomyocytes in ischemic myocardium. At the same time, CsA@PPTK obviously reduced the infarct size, fibrosis area and the protein expression of MMP-9, while increased the protein expression of CX43. Subsequently, the remodeling of the left ventricle was significant alleviated. Finally, heart function of MI/RI mice was markedly improved.

\section{Conclusion}

CsA@PPTK has great potential in the treatment of MI/RI. This study provides a novel class of heart protective biomimetic platform that is beneficial for treatment of MI/RI.

\section{Background}

Coronary heart disease such as blockage of coronary artery, acute myocardial infarction (AMI) and cardiac failure has become one of the leading causes of death in the world, accounting for over 17.3 million deaths every year [1]. Current therapy for AMI mainly concentrates on restoration coronary blood flow (reperfusion) through medications and/or revascularization procedures including via percutaneous coronary intervention or coronary artery bypass grafting. Although reperfusion strategy is vital for survival [2], patients with myocardial infarction remain an increased risk for myocardial ischemia/reperfusion injury (MI/RI), which currently lacks an effective therapeutic method [3-5]. So, exploring new strategies to treat $\mathrm{MI} / \mathrm{RI}$ shows important clinical significance and social benefits.

$\mathrm{MI} / \mathrm{RI}$ is the result of multiple factors, including apoptosis of cardiomyocytes, reactive oxygen species (ROS) burst production, and inflammatory cell recruitment. A single target based therapy is difficult to 
effectively inhibit reperfusion injury. During reperfusion, ROS bursts and $\mathrm{Ca}^{2+}$ overload occur in cardiomyocytes, leading to the opening of mitochondrial permeability transition pore (mPTP). The opening of MPTP further induces more ROS release from mitochondria and apoptosis of cardiomyocytes [6]. Apoptotic cardiomyocytes release damage associated molecular patterns (DAMP) and subsequently activate the inflammatory cascade reaction of endothelial cells. Then inflammatory cells were recruited to damaged myocardium tissue [7] and release ROS and cytokine in damaged myocardium tissue, which further aggravate apoptosis of cardiomyocytes and damage of myocardium. Finally, there forms a vicious cycle between burst release of ROS and apoptosis of cardiomyocytes in ischemic myocardium tissue.

Immunosuppressive regulatory $T$ cells (Tregs) account for about $5-10 \%$ of peripheral $C D 4^{+} T$ cells. Tregs participates in regulation of immune response in pathological or physiological states in an "active" manner, and plays an important role in maintaining immune tolerance and immune response homeostasis. Immunological studies have found that Tregs has protective effect on MI/RI[8]. Tregs can actively target to ischemic myocardium and play the role of anti-apoptosis, anti-inflammatory, antioxidant and reducing the remodeling of the left ventricle[9-12]. Therefore, many researchers hope to use adoptive input of Tregs or increase of Tregs to treat MI/RI[13]. However, the preparation of Tregs is difficult, and there are problems such as immune rejection if adoptive transfusion is carried out in clinical practice, which makes it difficult for Tregs to be applied in clinical practice. Nevertheless, Tregs internal effects suggest that scavenging ROS, reducing the opening of MPTP and alleviating cardiac inflammatory reaction are considered effective methods to improve myocardial function after MI/RI.

Cyclosporine A (CsA), an immunosuppressant, can inhibit the opening of mPTP through binding with cyclophilic protein D on the mitochondrial membrane[14]. However, the solubility of CsA in water is poor [15] and its distribution in vivo lacks ischemic myocardium-targeted characteristic. Previously, we reported that nanoparticle modified with SS-31 peptide increased the distribution of CsA in ischemic myocardium, and this nanoparticle inhibited apoptosis of cardiomyocytes through mitochondrial pathway[16]. Moreover, ROS is an important factor causing reperfusion injury, and it can damage a variety of cells in myocardial tissue through lipid peroxidation, DNA damage, etc. At the same time, ROS can also induce the recruitment of inflammatory cells, resulting in MI/RI [6]. Antioxidant drugs or bioactive materials can exert therapeutic effects on MI/RI by eliminating ROS [17]. In our previous study, we demonstrated that antioxidant drugs can play a role in the treatment of $\mathrm{MI} / \mathrm{RI}$ through the targeted delivery of resveratrol to ischemic myocardium. ROS-responsive materials can be reduced at high ROS levels, which can eliminate ROS in vivo while releasing drugs, consequently displayed antioxidant effect [18]. For example, polymer materials containing thioketal[19] and phenylboric acid[20] can play ROS scavenging role after being prepared as nanoparticles. However, most of drug delivery system is consisted of exogenous materials. When it enters the bloodstream, the mononuclear phagocytic system (MPS) can recognize these exogenous substances and quickly eliminate them from body, subsequently shortening their circulation time[21]. Thus, long-term retention in the blood and precise accumulation in 
ischemic myocardium are two major bottlenecks that ischemic myocardium targeting drug delivery system need to overcome.

In recent years, because biomimetic drug delivery system can not only simulate the function of cells or organelles in vivo, but also avoid the complex steps of cell or organelle preparation in vivo. Thus, biomimetic drug delivery system has achieved great development. Moreover, the composition of biomimetic drug delivery system is clear, and it has the prospect of clinical transformation. For example, some researchers have carried out biomimetic design of neutrophils[22], macrophages[23] and mitochondria[24] to treat diseases such as tumors or myocardial ischemia.

There are some biomacromolecule such as GPIB-IX-V, allBß3, intercellular cell adhesion molecule-1, pselectin and $\alpha 2 \beta 1$ integrin on the surface of platelet, which can bind with their corresponding receptors or collagens on vascular endothelial surface including von willebrand factor, aV $\beta 3$ integrin, $p$-selectin glycoprotein ligand (PSGL-1), collagen, etc. Besides, von willebrand factor is highly expressed on the endothelial cell surface of damaged blood vessels in ischemic myocardial tissue. Therefore, platelet displays damaged vascular endothelial targeting effect with the characteristic of multiple sites binding. Actually, the cardiac blood flow velocity is fast, the affinity of multiple sites binding of platelet with damaged blood vessels in ischemic myocardia is stronger than that of a single site binding drug delivery system $[25,26]$. This subsequently results in platelet recruitment in AMI area in vivo [1]. Thus, platelet and its membranes can be used as ischemic myocardium targeting material to guide nanoparticle to concentrate at ischemic myocardium $[1,27,28]$. Most importantly, when platelet membrane is coated on the surface of drug delivery system, due to CD47 on the membrane, it can not only specifically accumulate ischemic myocardium but also can effectively reduce the non-specific phagocytosis and prolong the circulation time of drug delivery system in vivo [29, 30]. Finally, the targeting effect to ischemic myocardium and biocompatibility of drug delivery system are greatly improved.

In this paper, poly (5,5-dimethyl-4,6-dithio-propylene glycol azelate) (PTK) was prepared. PTK contains a large amount of thioketal, which is not only sensitive to various types of $\mathrm{ROS}$ such as $\mathrm{O}^{2-}, \mathrm{ClO}^{-}$and $\mathrm{H}_{2} \mathrm{O}_{2}$ [31] but can also scavenge these ROS. CsA was encapsulated by PTK polymer to form CsA@PTK. The platelet membrane has the ischemic myocardium targeting effect, which can simulate the ischemic myocardium targeting effect of Tregs when platelet membrane is coated with nanoparticles. By using CsA@PTK as core and platelet membrane as shell, a Tregs biomimetic nanoparticle (CsA@PPTK) was prepared. CsA@PPTK could simulate Tregs to play a series of roles of Tregs such as ischemic myocardium targeting, anti-inflammatory, anti-apoptotic, scavenging of reactive oxygen species, and ultimately reducing the remodeling of the left ventricle and protecting ischemic myocardium (Scheme 1).

\section{Materials And Methods}

\section{Materials}


Cyclosporine A (CsA) was purchased from Shanghai Yuanye Biotechnology Co., Ltd (Shanghai, China). ROS test kit, mitochondrial membrane potential test kit (JC-1 solution) and RIPA lysate were purchased from Nanjing Beyotime Biotechnology Co. (Nanjing, China). Mitochondrial membrane permeability transition hole detection kit and mitochondrial superoxide red fluorescent probe were purchased from Shanghai Yisheng Biotechnology Co. (Shanghai, China). Antibodies against MMP-9, CX43, a-SMA and secondary antibodies were purchased from Servicebio (China). Antibodies against CD86 were purchased from Thermo invitrogen Inc. (USA). Antibodies against CD206 and FoxP3 were purchased from Cell Signaling Technology Inc. (USA). DAPI were purchased from Thermo Fisher Scientific Inc. (USA). TUNEL BrightRed Apoptosis Detection Kit was purchased from Servicebio (China). DiR dye was purchased from Ruitaibio (China). TNF-a, IL-1 $\beta$ ELISA kit were purchased from Servicebio (China). H9c2 cell line was bought from Shanghai Institute of Cell Biology (Shanghai, China). All chemical reagents were obtained from reagent suppliers. Kunming mice and SD rats were provided by the Experimental Animal Center of Air Force Medical University (Xi'an, China). Animal experiments were approved by the Air Force Medical University Institutional Animal Care and Utilization Committee (No: IACUC-20190505).

\section{Synthesis of 5,5-dimethyl-4,6-dithio-azelaic acid}

Acetone $(6.1 \mathrm{~mL})$ and 3-mercaptopropionic acid $(3.5 \mathrm{~mL})$ were mixed, and 3 drops of trifluoroacetic acid were added into above mixture. The reaction mixture was stirred overnight at room temperature. Next, the reaction mixture was cooled in ice bath for $4 \mathrm{~h}$, the crystals (5,5-dimethyl-4,6-dithio-azelaic acid, TK) were collected. The product was dried in a vacuum drying oven for $24 \mathrm{~h}$ at $36^{\circ} \mathrm{C}$. The structure of TK was identified by mass spectrometry (MS) and ${ }^{1} \mathrm{H}$ NMR.

\section{Synthesis of poly(5,5-dimethyl-4,6-dithio-propylene glycol azelate)}

1,3-Propanediol $(670 \mu \mathrm{L})$ and Sc trifluoromethyl sulfonate $(40 \mathrm{mg})$ were dissolved in $1 \mathrm{~mL}$ acetonitrile. The mixture was stirred at $40^{\circ} \mathrm{C}$ for $30 \mathrm{~min}$. Then, TK $(2 \mathrm{~g})$ was added into above mixture and stirred at $40{ }^{\circ} \mathrm{C}$ for $1 \mathrm{~h}$. Next, the reaction temperature was raised to $50^{\circ} \mathrm{C}$ under the protection of nitrogen. $1 \mathrm{~h}$ later, the temperature was raised to $90^{\circ} \mathrm{C}$ and the reaction mixture was stirred for $24 \mathrm{~h}$ under vacuum. After the temperature of reaction mixture decreased to $25^{\circ} \mathrm{C}$, chloroform $(1 \mathrm{~mL})$ and methanol $(8 \mathrm{~mL})$ were added into reaction mixture to precipitate product (poly(5,5-dimethyl-4,6-dithio-propylene glycol azelate), PTK). After being separated, the precipitation was dried in a vacuum at $37^{\circ} \mathrm{C}$ for $48 \mathrm{~h}$. The structure of PTK was identified by ${ }^{1} \mathrm{H}$ NMR and GPC.

\section{Preparation and Characterization of CsA@PPTK}

CsA@PTK nanoparticles were prepared by emulsion evaporation method. Briefly, PTK (10 mg) and CsA (2 $\mathrm{mg}$ ) were dispersed into $20 \mathrm{~mL}$ polyvinyl alcohol (PVA, 0.5\%) solution containing $2 \mathrm{~mL}$ dichloromethane. After mixture was performed ultrasound at $270 \mathrm{~W}$ for $3 \mathrm{~min}$, the dichloromethane was removed by stirring the mixture for $12 \mathrm{~h}$ at room temperature. The nanoparticle solution was filtered by using $0.4 \mu \mathrm{m}$ membrane to remove free CsA. Ultrafiltration centrifuge tube (Millipore, USA) was used to remove PVA. Finally, CsA@PTK powder was obtained by lyophilization. Blank nanoparticle without CsA (@PTK) was 
prepared by the same method. Coumarin 6 labeled CsA@PTK (Coumarin 6@PTK) and Cy7.5 labeled CsA@PTK (Cy7.5@PTK) was prepared by using the same method as preparation for CsA@PTK except for adding Coumarin 6 (final concentration was $1 \mathrm{mg} / \mathrm{mL}$ ) or Cy 7.5 (final concentration was $0.5 \mathrm{mg} / \mathrm{mL}$ ) into dichloromethane solution.

The platelets were isolated from rat plasma by differential centrifugation, and the platelet membrane was derived by using a repeated freeze-thaw process[32]. By using repeated extrusion method with platelet membrane[33], CsA@PPTK, @PPTK, Coumarin6@PPTK and Cy7.5@PPTK were prepared by using CsA@PTK, @PTK, Coumarin6@PTK and Cy7.5@PTK as inner core, respectively. Size and zeta potential of nanoparticle were measured by using a Malvern ZEN 3600 Zetasizer. Hitachi S-4800 transmission electron microscope (TEM) was used to visualize the shape of nanoparticle. The protein on CsA@PPTK surface was studied by SDS-PAGE. The hemolytic effect of CsA@PPTK was investigated by using rat blood red cell.

\section{In vitro CsA release}

Dialysis bags (MWCO: 3500 Da, Millipore) containing $5 \mathrm{~mL}$ suspension solution of different formulations were respectively immersed into $500 \mathrm{~mL}$ of PBS (pH 7.4) or NaClO-containing PBS solution under horizontal shaking at $37^{\circ} \mathrm{C} .50 \mu \mathrm{L}$ solution in dialysis bag was taken out at different time point. CsA in release medium was analyzed by high performance liquid chromatography (HPLC, 2695/2996, Waters Corporation). The Dikma $\mathrm{C}_{18}$ column $(4.6 \mathrm{~mm} \times 250 \mathrm{~mm}, 5 \mu \mathrm{m})$ was used to separate the CsA. Mobile phase is consisted with acetonitrile and water ( $\mathrm{v}: \mathrm{v}=90: 10)$. The column temperature was $55^{\circ} \mathrm{C}$. The flow rate was $1 \mathrm{~mL} / \mathrm{min}$. The detection wavelength was $210 \mathrm{~nm}$. The equation of the standard curve is $y=560.39 x-288.15, R^{2}=0.9999$.

\section{Hypoxia re-oxygenation damaged $\mathrm{H} 9 \mathrm{c} 2$ cells model}

To prepare hypoxic culture solution, sodium lactate $(1.12 \mathrm{~g})$, 4-hydroxyethylpiperazine ethane sulfonic acid $(0.475 \mathrm{~g})$, sodium chloride $(4.007 \mathrm{~g})$, 2-deoxy-D-glucose $(0.82 \mathrm{~g})$, potassium chloride $(0.59 \mathrm{~g})$, sodium sulfate $(0.093 \mathrm{~g})$, hydrated calcium chloride $(0.065 \mathrm{~g})$ and magnesium chloride $(0.05 \mathrm{~g})$ were dissolved into $500 \mathrm{~mL}$ deionized water.

To set up hypoxia re-oxygenation $(\mathrm{H} / \mathrm{R})$ injured $\mathrm{H} 9 \mathrm{c} 2$ cells model, $\mathrm{H} 9 \mathrm{c} 2$ cells were incubated in a hypoxic environment $\left(95 \% \mathrm{~N}_{2}\right.$ and $\left.5 \% \mathrm{CO}_{2}\right)$ for $3 \mathrm{~h}$ at $37^{\circ} \mathrm{C}$ by using hypoxic culture medium. Next, the culture medium was replaced with DMEM solution containing CsA, CsA@PTK and CsA@PPTK, and H9c2 cells were cultured in a standard incubator with $5 \% \mathrm{CO}_{2}$ in normal atmosphere for $4 \mathrm{~h}$ at $37^{\circ} \mathrm{C}$. Then, the cell viability, intracellular ROS, mitochondrial ROS, the opening of MPTP and mitochondrial membrane potential of H9c2 cells were investigated.

\section{The effect of CsA@PPTK on the viability of H/R damaged H9c2 cells}


H9c2 cells were treated with MTT $(20 \mu \mathrm{L} /$ well, $5 \mathrm{mg} / \mathrm{mL})$ for $4 \mathrm{~h}$. The cell culture medium was replaced with dimethylsulfoxide (DMSO) solution. By using a microplate reader (Bio-Rad Laboratories, Richmond, CA, USA), the absorbance of DMSO solution was detected at $490 \mathrm{~nm}$. The control cells were cultured in normoxic conditions with FBS-free DMEM solution for $7 \mathrm{~h}$.

\section{The effect of CsA@PPTK on the intracellular ROS of H/R damaged H9c2 cells}

The intracellular ROS was detected by using 2',7'-dichlorodihydrofluorescin diacetate (DCFH-DA). DCFHDA probe was diluted with serum-free medium at the ratio of 1:1000. Then, DCFH-DA solution was cultured with $\mathrm{H} / \mathrm{R}$ damaged $\mathrm{H} 9 \mathrm{c} 2$ cells for 20 min at $37^{\circ} \mathrm{C}$. After DCFH-DA solution was removed, and the cells were washed with serum-free culture medium for 3 times. DAPI was used for nucleus staining. The cells were observed under fluorescent microscope (Olympus, Japan). The fluorescence intensity was semi-quantified with Image Pro software. Fluorescence intensity indicated ROS level in the cell. The stronger the fluorescence intensity was, the higher the ROS level was.

\section{The effect of CsA@PPTK on the mitochondrial ROS of H/R damaged H9c2 cells}

MitoSOX Indicator was diluted to $5 \mu \mathrm{M}$ with HBSS. Then, the diluted MitoSOX Indicator solution was cultured with $\mathrm{H} / \mathrm{R}$ damaged $\mathrm{H} 9 \mathrm{c} 2$ cells for 10 min at $37^{\circ} \mathrm{C}$. DAPI was used for nucleus staining. The cells were observed by using confocal laser scanning microscopy (CLSM, Olympus, Japan). The fluorescence intensity was semi-quantified with Image Pro software. Fluorescence intensity indicated ROS level in mitochondria. The stronger the fluorescence intensity was, the higher the ROS level was.

\section{The effect of CsA@PPTK on the opening of mPTP of H/R damaged H9c2 cells}

$\mathrm{H} / \mathrm{R}$ damaged $\mathrm{H} 9 \mathrm{c} 2$ cells were incubated with $5 \mu \mathrm{L}$ Calcein $\mathrm{AM}$ solution $(1 \mathrm{mmol} / \mathrm{L})$ and $5 \mu \mathrm{L} \mathrm{CoCl}{ }_{2}$ solution $(80 \mathrm{mM})$ for $15 \mathrm{~min}$ at $37^{\circ} \mathrm{C}$. Next, HBSS/Ca $(3.5 \mathrm{~mL})$ was added into cell mixture. The cells were collected and re-suspended in buffer solution $(400 \mu \mathrm{L})$ to carry out flow cytometric analysis. The stronger the fluorescence intensity was, the less the opening of MPTP was.

\section{The effect of CsA@PPTK on the mitochondrial membrane potential of H/R damaged H9c2 cells}

JC-1 solution $(5 \mu \mathrm{g} / \mathrm{mL})$ was cultured with $\mathrm{H} / \mathrm{R}$ damaged $\mathrm{H} 9 \mathrm{c} 2$ cells for 15 min at $37^{\circ} \mathrm{C}$. Then, the $\mathrm{H} 9 \mathrm{c} 2$ cells were slightly rinsed 2 times with assay buffer. The fluorescent intensity of $\mathrm{H} 9 \mathrm{c} 2$ cell solution were respectively detected at 530/590 nm (excitation/emission wavelength, red fluorescence) and 485/530 nm (green fluorescence) by fluorescence spectrophotometer. The ratio between red fluorescent intensity and green fluorescent intensity was calculated, which indicated the mitochondrial membrane potential. The greater the ratio value was, the higher the mitochondrial membrane potential was.

\section{Cellular uptake of CsA@PPTK on H/R damaged H9c2 cells}

H9c2 cells were seeded into 6 well plates $\left(1 \times 10^{5}\right.$ cells/well). After hypoxia for 3 h, H9c2 cells were incubated with DMEM solution containing CsA, CsA@PTK or CsA@PPTK $(30 \mu \mathrm{g} \mathrm{CsA} / \mathrm{mL})$ in a standard 
incubator with $5 \% \mathrm{CO}_{2}$ in normal atmosphere for $0.5 \mathrm{~h}$ and $2 \mathrm{~h}$. $\mathrm{H} 9 \mathrm{c} 2$ cells were washed for 3 times with PBS (pH 7.4) and lysed by $100 \mu \mathrm{L}$ RIPA lysis buffer. CsA in cell lysis was determined by HPLC. The protein content in cell lysis was determined by coomassie brilliant blue. The content of CsA in cell lysis was normalized by protein content in cell lysis.

To investigate the endocytic pathway of CsA@PPTK, H9c2 cells were seeded into 6 well plates $\left(1 \times 10^{5}\right.$ cells/well). After hypoxia for $2 \mathrm{~h}$, 2-deoxy-D-glucosesucrose (ATP depletion agent, $1 \mathrm{mg} / \mathrm{mL}$ ), sucrose (inhibitor of clathrin-mediated uptake, $150 \mathrm{mg} / \mathrm{mL}$ ), methyl- $\beta$-cyclodextrin (inhibitor of caveolae-mediated uptake, $0.005 \mathrm{mg} / \mathrm{mL}$ ), colchicine (inhibitor of macropinocytosis, $0.8 \mathrm{mg} / \mathrm{mL}$ ) were respectively added to $\mathrm{H} 9 \mathrm{c} 2$ cells, and $\mathrm{H} 9 \mathrm{c} 2$ cells were incubated for $1 \mathrm{~h}$ at $37^{\circ} \mathrm{C}$ in hypoxic culture medium. Then, the hypoxic culture medium was replaced with DMEM solution containing CsA, CsA@PTK or CsA@PPTK (30 $\mu \mathrm{g}$ $\mathrm{CsA} / \mathrm{mL}$ ), and $\mathrm{H} 9 \mathrm{c} 2$ cells were incubated in a standard incubator with $5 \% \mathrm{CO}_{2}$ in normal atmosphere for 2 h. The cells were collected and lysed. Finally, CsA in cell lysis was determined by using the same method as described above.

\section{MI/RI mice model}

Kunming mice were anesthetized with $2 \%$ isoflurane inhalation. About $2 \mathrm{~mm}$ long vertical incision was cut at 2-3 mm away from the left sternal border. The chest wall muscle was separated. A small hole was made at intercostal space with a small hemostatic forceps to open the pleural membrane and pericardium. With the slightly open of hemostatic forceps, the heart was smoothly exposed out of the hole. By using a 6/0 silk suture, the left anterior descending coronary artery (LDA) was ligated at a site 3 $\mathrm{mm}$ from its origin. Only when the anterior wall of left ventricle (LV) became pale, the ligation was regarded as success. Heart was put back into chest immediately after ligation. The air in chest was extruded out, and the chest was closed with $4 / 0$ suture. 30 min later, the $6 / 0$ suture was carefully pulled out to restore the blood flow of LDA. The sham group performed the same surgical procedure except that LDA was not ligated.

\section{Biodistribution of CsA@PPTK in MI/RI mice}

Acute myocardial ischemic Kunming mice were intravenously injected a single dose of Cy7.5 labeled CsA@PPTK (Cy7.5@PPTK) 5 min before reperfusion. 24 h after injection of Cy7.5@PPTK, various organs (heart, liver, lung, spleen, and kidney) were collected and imaged using Caliper IVIS Lumina $₫$ (Siemens, Germany). The heart tissue was then cut into five or six transverse sections and imaged again.

\section{Cardiac function of MI/RI mice}

The cardiac function of MI/RI mice was assessed by transthoracic echocardiogram (Vevo 2100, VisualSonics, Canada). Animals were anesthetized using a mixture of isoflurane and oxygen before undergoing transthoracic echocardiogram procedure at the 4 week and 10 week after reperfusion. Two dimensional images of LV were collected. Then, LV functional parameters such as left ventricular 
shortening fraction (FS \%) and left ventricular ejection fraction (LVEF \%) were calculated by using Vevo 2100 softwar.

\section{Histological and immunohistochemical analysis}

$\mathrm{MI} / \mathrm{RI}$ mice were sacrificed by intraperitoneal injection of over-dose pentobarbital sodium. Hearts and blood were harvested. The contents of inflammatory cytokines such as IL-1 $\beta$ and TGF- $\beta$ in plasma were determined by ELISA kit. After being fixed in $4 \%$ paraformaldehyde, heart tissue was dehydrated by using gradient concentration of alcohol and embedded into paraffin. Then, heart tissue was cut into $4 \mu \mathrm{m}$ thick sections for histological and immunohistological staining. For infarct size and fibrosis evaluation, masson trichrome staining was performed and total sections were scanned to acquire the whole images of heart horizontal planes. The fibrotic area was identified using ImageJ software. The fibrosis was calculated as fibrotic area/total area in images. Tregs cell in section of heart tissues were marked by FoxP3 antibody. M1-type macrophage and M2-type macrophage in section of heart tissues were tracked by CD86 antibody and CD206 antibody, respective. Then, fluorescence of FoxP3, CD206 and CD86 in section of heart tissues was observed by using CLSM. The expression of MMP-9, CX43 and a-SMA in section of heart tissues was observed by immunofluorescence method. TUNEL staining was used to track the apoptotic cardiomyocyte.

\section{Statistical analysis}

All results are expressed as means $\pm S D$. Comparison between two groups was performed with two-tailed Student's $t$ test. The difference was considered statistically significant at the value of $p<0.05,0.01$ and 0.001 .

\section{Results}

\section{Characterization of TK and PTK}

The mass spectrum of 5,5-dimethyl-4,6-dithio-azelaic acid (TK) is showing in Figure.S1A, which was consistent with the theoretic molecular weight of TK. In addition, ${ }^{1} \mathrm{H}$ NMR spectroscopy of TK showed peaks at 1.59, 2.90 and 2.68 ppm, it was assigned to $\mathrm{Ha}, \mathrm{Hb}$ and $\mathrm{Hc}$ of TK, respectively (Figure.S1B). Compared with TK, there was two new peak appeared at 4.18 and $1.98 \mathrm{ppm}$ in ${ }^{1} \mathrm{H}$ NMR spectroscopy of poly(5,5-dimethyl-4,6-dithio-propylene glycol azelate) (PTK), which were assigned to $\mathrm{Hd}$ and $\mathrm{He}$ of propanediol (Figure.S2). The molecular weight of PTK was 20505 Da determined by gel penetration chromatography (Figure.S3).

\section{Characterization of CsA@PPTK}

Transmission electron microscopy showed that the appearance of CsA@PTK and CsA@PPTK was spherical with good dispersion (Figure.1A). Besides, CsA@PTK displayed a uniform structure from inside to outside, while CsA@PPTK showed obvious core-shell structure. This indicated that platelet membrane 
was coated on the surface of CsA@PTK. As compared to CsA@PTK, the size of CsA@PPTK did not show significant change. The zeta potential of CsA@PPTK obviously decreased to - $25 \mathrm{Mv}$, which was similar to zeta potential of platelet (Figure.1B). The co-localization of the DiR-labeled platelet membrane (red) and coumarin 6 labeled CsA@PTK (green) further substantiated the successful coating of platelet membrane on the surface of CSA@PTK (Figure.1C). SDS-PAGE electrophoresis revealed that the majority protein of platelet membrane was retained in CsA@PPTK (Figure.1D). All above results indicated that the platelet membrane was coated on PTK nanoparticles successfully. The drug loading of CsA in CsA@PPTK was 4.97\%. As shown in Figure.1E, the size and PDI of CsA@PPTK kept stable over 23 days, indicating platelet membrane coating increased the stability of CsA@PPTK. Moreover, CsA@PTK and CsA@PPTK did not cause significant hemolysis at concentration of $1 \mathrm{mg} / \mathrm{mL}$.

\section{ROS scavenging ability and in vitro drug release of CsA@PPTK}

When CsA@PTK and CsA@PPTK was suspended in PBS, the suspension solution remained opalescence for 24 h. However, after CsA@PTK and CsA@PPTK was suspended in PBS solution containing 1 mM sodium hypochlorite ( $\mathrm{NaClO}$, an important $\mathrm{ROS}$ generated in ischemic myocardium under oxidative stress), CsA@PTK and CsA@PPTK suspension solutions became clear within 12 h. In PBS solution containing 10 mM NaClO, CsA@PTK and CsA@PPTK suspension solutions became clear within $3 \mathrm{~h}$ (Figure.2A, Figure.S4A). These results indicated that degradation rate of CsA@PTK and CsA@PPTK was accelerated with the increase of $\mathrm{NaClO}$ concentration. The degradation of CsA@PTK and CsA@PPTK exhibited ROS-dependent manner. The drug release tests indicated that after incubation with PBS solution containing $1 \mathrm{mM} \mathrm{NaClO}$ at $37{ }^{\circ} \mathrm{C}$ for $4 \mathrm{~h}$, about 70\% CsA was released from CsA@PTK and CsA@PPTK. When concentration of $\mathrm{NaClO}$ in PBS solution increased to $10 \mathrm{mM}, 95 \%$ CsA was released from CsA@PTK and CsA@PPTK within 4 h. Nevertheless, when CsA@PTK or CsA@PPTK was incubated with PBS, less than 70\% CsA was released within $24 \mathrm{~h}$ (Figure.2B, Figure.S4B). These results demonstrated that platelet membrane coating did not affect the ROS-responsive characteristic of CsA@PPTK. CsA@PTK and CsA@PPTK released CsA in ROS-dependent manner. The high ROS level microenvironment induced by ischemia reperfusion would facilitate the release of CsA from CsA@PPTK.

The in vitro ROS scavengingproperty of CsA@PPTK was assessed by DPPH assay[34]. When @PPTK, CsA@PTK and CsA@PPTK was incubated with DPPH solution for 15 min, the dark purple color of DPPH solution apparently faded as compared with DPPH or DPPH/CsA ethanol solution (Figure.2C).

Quantitative analysis showed that the absorbance value of DPPH solution containing @PPTK, CsA@PTK and CsA@PPTK significantly decreased with the prolongation of incubation time (Figure.2D), revealing the clearance effect of @PPTK, CsA@PTK and CsA@PPTK on DPPH free radical.

\section{The protective effectof CsA@PPTK on H/R injured H9c2 cells}

At first, the cytotoxicity of PPTK on cardiomyocytes was assessed by MTT method. As shown in Figure.S5A, 0.2 1 mg/mL @PTK increased the activity of normal H9c2 cells after incubation for $24 \mathrm{~h}$. In addition, $0.5 \mathrm{mg} / \mathrm{mL}, 1 \mathrm{mg} / \mathrm{mL}$ and $2 \mathrm{mg} / \mathrm{mL}$ @PTK improved the activity of hypoxia for $3 \mathrm{~h}$ and reoxygenation for $4 \mathrm{~h}(\mathrm{H} / \mathrm{R})$ injured $\mathrm{H} 9 \mathrm{c} 2$ cells (Figure.S5B). It was reported that the optimized 
concentration of CsA to protect $\mathrm{H} / \mathrm{R}$ injured $\mathrm{H} 9 \mathrm{c} 2$ cells was 15 and $30 \mu \mathrm{g} / \mathrm{mL}$ [16]. The drug loading of CsA@PPTK was 4.97\%. Therefore, 1 mg/mL @PTK was much higher than the maximum dose of @PTK that needed in the experiment, indicating that under the therapeutic doses, CsA@PTK exhibited no cytotoxicity on cardiomyocytes.

As shown in Figure.2E, CsA, CsA@PTK and CsA@PPTK improved the activity of H/R injured H9c2 cells. There was no significant difference in activity of $H / R$ injured $H 9 c 2$ cells between $15 \mu \mathrm{g} / \mathrm{mL}$ and $30 \mu \mathrm{g} / \mathrm{mL}$ free CsA. The protective effect of CsA@PTK and CsA@PPTK on H/R injured H9c2 cells was similar to CsA when concentration of CsA was $15 \mu \mathrm{g} / \mathrm{mL}$. However, when the concentration of CsA was $30 \mu \mathrm{g} / \mathrm{mL}$, the protective effect of CsA@PTK and CsA@PPTK on H/R injured H9c2 cells was significantly stronger than that of CsA. This resulted from two factors. On the one hand, CsA@PPTK and CsA@PTK increased solubility of CsA. On the other hand, CsA@PPTK and CsA@PTK could scavenge ROS in H9c2 cells induced by $\mathrm{H} / \mathrm{R}$.

\section{The protective effect of CsA@PPTK on mitochondria of H/R injured H9c2 cells}

DCFH-DA staining demonstrated that there were a large amount of ROS in no-treated H/R injured H9c2 cells, indicating acute oxidative stress occurred after H/R (Figure.S6A). However, compared with notreated H9c2 cells, ROS-positive cells and ROS level were significantly reduced in @PTK, CsA@PTK and CsA@PPTK treated H/R injured H9c2 cells. Besides, there were no significant difference in ROS level between @PTK treated and CsA@PTK treated H/R injured H9c2 cells (Figure.S6B). The above results suggested that ROS level were significantly inhibited by @PTK, CsA@PTK and CsA@PPTK in H/R injured $\mathrm{H} 9 \mathrm{c} 2$ cells, and PTK exerted an important role in protection $\mathrm{H} 9 \mathrm{c} 2$ cells from $\mathrm{H} / \mathrm{R}$ injury by reducing ROS level.

The effect of CsA@PPTK on mitochondrial ROS is showing in Figure.3A-B, compared with control group, red fluorescence intensity was much stronger in $\mathrm{H} / \mathrm{R}$ injured $\mathrm{H} 9 \mathrm{c} 2$ cells, while red fluorescence intensity was much weaker in CsA, @PPTK, CsA@PTK and CsA@PPTK treated H/R injured H9c2 cells. The statistical results of red fluorescence intensity in $\mathrm{H} / \mathrm{R}$ injured $\mathrm{H} 9 \mathrm{c} 2$ cells are showing in Figure.3C. The ratio of red to blue fluorescence intensity in $\mathrm{H} / \mathrm{R}$ model group was significantly higher than that in control group, indicating that mitochondria produced a large amount of ROS after H9c2 cells was injured by H/R. Compared with H/R model group, the ratio of red to blue fluorescence intensity in CsA, @PPTK, CsA@PTK and CsA@PPTK treated group was significantly reduced. Meanwhile, CsA@PPTK treated H/R injured H9c2 cells showed the lowest ratio of red to blue fluorescence intensity, indicating CsA, @PPTK, CsA@PTK and CsA@PPTK reduced mitochondrial ROS level in H/R injured H9c2 cells, and CsA@PPTK exhibited the strongest effect on reducing mitochondrial ROS level in H/R injured H9c2 cells.

The effect of CsA@PPTK on the opening of mPTP in H/R injured H9c2 cells was shown in Figure.3D-E. Compared with the normal group, the calcein fluorescence intensity in mitochondria in H/R injured H9c2 cells was significantly decreased, indicating that $H / R$ could significantly increase the opening of $m P T P$ in $\mathrm{H} 9 \mathrm{c} 2$ cells. Compared with the H/R model group, the calcein fluorescence intensity in mitochondria was significantly increased in CsA, CsA@PTK and CsA@PPTK treated H/R injured H9c2 cells, indicating that 
CsA, CsA@PTK and CsA@PPTK played a protective role against H/R injury by reducing the opening of mPTP in H/R injured H9c2 cells.

Next, JC-1 staining was used to detect the mitochondrial membrane potential, and the results are showing in Figure.3F. Compared with normal cultured H9c2 cells, the red fluorescence was obviously decreased and green fluorescence was obviously increased in H/R injured H9c2 cells, suggesting mitochondrial membrane potential was damaged during $\mathrm{H} / \mathrm{R}$ in $\mathrm{H} 9 \mathrm{c} 2$ cells. However, compared with $\mathrm{H} / \mathrm{R}$ model group, the ratio of red to green fluorescence was significantly increased in CsA, CsA@PTK and CsA@PPTK treated H/R injured H9c2 cells, indicating that CsA, CsA@PTK and CsA@PPTK recovered the damaged mitochondrial membrane potential.

\section{Cellular uptake of CsA@PPTK by H/R injured H9c2 cells}

The specific cellular uptake behavior of H9c2 cells towards CsA@PPTKwas studied by HPLC. The uptake of CsA@PPTK by H9c2 cells is shown in Figure.S7. The uptake of CsA@PTK and CsA@PPTK by normal and H/R injured H9c2 cells exhibited time-dependent manner. The uptake of CsA@PPTK in H/R injured $\mathrm{H} 9 \mathrm{c} 2$ cells was much higher than that in normal H9c2 cells at $2 \mathrm{~h}$. Compared with CsA@PTK, a large amount of CsA@PPTK was taken up by H/R injured H9c2 cells.

The effects of different uptake pathway inhibitors on the uptake of CsA@PPTK in H/R injured H9c2 cells were observed. As shown in Figure.S8, sucrose, colchicine, 2-deoxy-D-glucose and methyl- $\beta$-cyclodextrin showed no significant effect on the uptake of CsA@PPTK by H/R injured H9c2 cells. This suggested that the receptor-mediated endocytosis pathway did not involve in the uptake of CsA@PPTK by H/R injured H9c2 cells. Compared with the control group, the uptake of CsA@PPTK in H/R injured H9c2 cells significantly reduced at $4{ }^{\circ} \mathrm{C}$. In theory, adsorptive endocytosis is temperature dependent. The platelet membrane coated on the surface of CsA@PPTK is very similar with membrane of H/R injured H9c2 cells, which resulted in the strong adsorptive effect between CsA@PPTK and H/R injured H9c2 cells. Therefore, CsA@PPTK was taken up by H/R injured H9c2 cells mainly through adsorption endocytosis[35].

\section{The ex-vivo targeted of CsA@PPTK}

The specific binding of Coumarin 6@PPTK on aortic vessels with endothelia injured is showing in Figure.4A-B. The green fluorescence intensity of Coumarin 6@PPTK in normal aortic vessels was very weak, indicating little amount of Coumarin6@PPTK was bound with normal aortic vessels. Compared with Coumarin6@PTK, the green fluorescence intensity of Coumarin 6@PPTK in aortic vessels with endothelia injured was much stronger, suggesting a large amount of Coumarin 6@PPTK was bound with endothelia injured aortic vessels. The above data implied that CsA@PPTK could specifically bind with endothelia injured vessels in ischemic area.

\section{In vivo targeting of CsA@PPTK}

As shown in Figure.S9, after acute myocardial ischemic mice were intravenously injected a single dose of Cy7.5 labeled CsA@PPTK (Cy7.5@PPTK) 5 min before reperfusion, Cy7.5 labeled CsA@PTK 
(Cy7.5@PTK) was mainly distributed in the liver and spleen, and little amount of Cy7.5@PTK was distributed in heart. However, compared with Cy7.5@PTK, a large amount of Cy7.5@PPTK was distributed in the heart, while little amount of Cy7.5@PPTK was distributed in the liver of MI/RI mice. As shown in Figure.4C, Cy7.5@PPTK was distributed in the whole heart when it was intravenously injected to sham mice. However, Cy7.5@PPTK was mainly distributed in downstream areas of the occluded coronary artery heart when it was intravenously injected to MI/RI mice. Cy7.5@PTK distributed in the whole heart of MI/RI mice. Next, the heart was cut into six or seven transverse sections from apex to atrium, and the distribution of Cy7.5@PPTK in transverse sections is showing in Figure.4D. Cy7.5@PPTK distributed in all heart transverse sections of sham mice. Cy7.5@PTK also appeared in all heart transverse sections of $\mathrm{MI} / \mathrm{RI}$ mice. However, Cy7.5@PPTK was mainly distributed in transverse sections of ischemic area especial in transverse section of apex of MI/RI mice heart. This indicated that Cy7.5@PPTK exhibited obvious ischemic myocardium targeting characteristic.

\section{In vivo therapeutic effect of CsA@PPTK on MI/RI mice}

On 28th day after reperfusion, echocardiography was applied to reveal the recovery of overall cardiac functions of $\mathrm{Ml} / \mathrm{RI}$ mice, and results are showing in Figure.5. The mice in sham group showed regular and stable heart beats along with a large amplitude. The amplitude of heart beats was reduced and ventricular cavity volume was increased in normal saline treated MI/RI mice (Figure.5A), suggesting an abnormal ventricular remodeling occurred. CsA and its preparations increased the amplitude of heart beats and decrease the ventricular volume of MI/RI mice as compared to normal saline. The left ventricular ejection fraction (LVEF) and fractional shortening (FS) reveal systolic function of the heart. Quantitative analysis revealed that EF and FS increased along the sequence of normal saline, CsA, @PPTK, CsA@PTK and CsA@PPTK in MI/RI mice (Figure.5B-C), manifesting the improvement of systolic function and inhibition of abnormal ventricular remodeling by using CsA, @PPTK, CsA@PTK and CsA@PPTK. Compared with normal saline, @PPTK obviously increased the heart function of MI/RI mice, indicating that ROS scavenging was an effective and necessary treatment method for MI/RI mice. CsA@PPTK treated mice showed the highest EF and FS in all group, signifying that ROS scavenging and anti-apoptosis could improve heart function significantly. The echocardiography was also applied to reveal the recovery of overall cardiac functions on 70th day post MI/RI, and results showed that CsA@PPTK performed the strongest effect on the recovery of heart function at dose of $2.5 \mathrm{mg} / \mathrm{kg}$ (Figure.S10).This suggested that early reduction of reperfusion injury increased cardiac function for a longer period of time.

Figure.S11 shows the ROS level in infarcted heart area 1 day after reperfusion, red fluorescence represents ROS. The results indicated that compared with sham group, the ROS level in ischemic myocardium in normal saline treated group (MI/RI model group) was significantly increased. CsA, @PPTK, CsA@PTK and CsA@PPTK markedly decreased ROS level in ischemic myocardium. CsA@PPTK treated group showed the lowest ROS level in ischemic myocardium among 4 groups, which resulted from the following two reasons. Firstly, CsA inhibited the production of ROS in mitochondria. Secondly, PTK strongly scavenged ROS in ischemic cardiomyocytes and ischemic myocardium. 
The phenotype of macrophage in the infarcted heart tissue was detected on 4th day post reperfusion. The M1 and M2 type macrophages were stained by their surface markers of CD86 (red) and CD206 (green), respectively. As shown in Figure.6A-B, the heart tissue exhibited strong green fluorescence in CsA@PPTK treated group, while heart tissue displayed weak green fluorescence in normal saline treated group. As compared with normal saline treated group, the ratio of $\mathrm{M} 2$ type macrophages to $\mathrm{M} 1$ type macrophages obviously increased in heart tissue in CsA, CsA@PTK, @PPTK and CsA@PPTK treated groups. CsA@PPTK treated group exhibited the highest ratio of M2 type macrophages to M1 type macrophages in heart tissue among 4 groups. Besides, as compared with normal saline treated group, the number of $M 2$ type macrophages and $M 1$ type macrophages significantly reduced in heart tissue in CsA, CsA@PTK, @PPTK and CsA@PPTK treated groups. CsA@PPTK treated groups showed a minimum of $\mathrm{M} 2$ type macrophages and $\mathrm{M} 1$ type macrophages in all groups. In addition, acute inflammatory reaction usually occurs after reperfusion, which is reflected by the secretion of inflammatory factors such as TNF-a from the activated immune cells [36]. The experimental results showed that compared with normal saline treated group, IL-1 $\beta$ and TGF- $\beta$ concentration in serum was significantly decreased in CsA@PPTK treated MI/RI mice on 4th day after reperfusion (Figure.6C-D). Immunofluorescence staining results showed that compared with the sham group, Tregs in myocardial tissue significantly increased in the model group, and Tregs in myocardial tissue decreased after the treatment of CsA. Compared with model group, CsA@PPTK significantly increased the number of Tregs in the infarct area (Figure.6E-F). The above data demonstrated that CsA@PPTK alleviated MI/RI and recovered the heart function through regulating the phenotype of macrophage and reducing systemic inflammatory reaction.

TUNEL staining images of infarcted heart area on 7th day after reperfusion is showing in Figure.7A-B. The positive staining represents the apoptotic cardiomyocytes. Compared with normal saline treatment, CsA, @PPTK, CsA@PTK and CsA@PPTK significantly reduced the apoptosis of cardiomyocytes in ischemic area. CsA@PPTK treated group displayed a minimum number of apoptotic cardiomyocytes in ischemic area among all groups, which resulted from inhibiting the production of mitochondrial ROS and the recovery of damaged mitochondrial membrane potential of cardiomyocytes.

Masson trichrome staining and H\&E staining of heart tissue are showing in Figure.7C. In masson trichrome staining section, normal heart muscle is represented in red and fibrotic areas are represented in blue. Compared with sham group, the blue areas were increased in normal saline treatment group. Meanwhile, the ventricles were significantly dilated, and the walls of the ventricles were significantly thinner in the model group than that in sham group. The percentage of myocardial fibrosis area in myocardial section area of mice is showing in Figure.7D. The area of myocardial fibrosis in normal saline group was significant greater than that in sham group. CsA, @PPTK, CsA@PTK and CsA@PPTK significantly reduced myocardial fibrosis as compared with normal saline. As compared with the same dose of CsA, CsA@PPTK significantly reduced myocardial fibrosis at the dose of $2.5 \mathrm{mg} / \mathrm{kg}$. The percentage of left ventricular area to myocardial section area is showing in Figure.7E. The percentage of left ventricular area to myocardial section area in normal saline group was marked smaller than that in sham group. CsA@PPTK significantly increased the percentage of left ventricular area to myocardial section area as compared with normal saline. As compared with the same dose of CsA, CsA@PPTK 
significantly increased the percentage of left ventricular area to myocardial section area at the dose of $2.5 \mathrm{mg} / \mathrm{kg}$. The above results demonstrated that CsA@PPTK strongly inhibited the cardiac remodeling and fibrosis, subsequently markedly improved the cardiac function of MI/RI mice. These results were also consistent with the echocardiography data. In addition, a large number of inflammatory cells were infiltrated, and the structure of cardiomyocytes was unclear in normal saline group. The cardiomyocytes in sham group were orderly arranged and the cell structure was intact. In $2.5 \mathrm{mg} / \mathrm{kg}$ CsA@PPTK treatment group, the arrangement of cardiomyocytes was regular, and the inflammatory cell infiltration was decreased. These results indicated that CsA@PPTK exhibited anti-inflammatory effect and protected myocardium through anti-inflammatory effect.

The CX43 is the main protein that constitutes the intercellular gap junction of ventricular myocytes[37]. The effect of CsA@PPTK on the expression of CX43 in myocardium of MI/RI mice is showing in Figure.8A-B. Compared with sham group, the expression of CX43 in the anterior wall of the left ventricle was significantly decreased in normal saline group. Compared with normal saline and same dose of CsA@PTK, CsA@PPTK significantly increased the expression of CX43 in the anterior wall of the left ventricle. These results indicated that CsA@PPTK significantly protected the activity of cardiomyocytes after $\mathrm{MI} / \mathrm{RI}$, and subsequently improved the cardiac function of MI/RI mice.

The effect of CsA@PPTK on the expression of MMP-9 in myocardium of MI/RI mice is showing in Figure.8C-D. Compared with sham group, the expression of MMP-9 in the left ventricle anterior wall was significantly increased in normal saline group. CsA, @PPTK, CsA@PTK and CsA@PPTK significantly reduced the expression of MMP-9 in the left ventricle anterior wall of MI/RI mice as compared with normal saline. CsA@PPTK markedly reduced the expression of MMP-9 in the left ventricle anterior wall as compared with the same dose of CsA@PTK, suggesting CsA@PPTK effectively protected heart from ventricular remodeling.

Interestingly, the arteriole density in the infarct area was also increased (Figure.8E) in CsA@PPTK treatment group, indicating that blood vessels were preserved in infarct area by using CsA@PPTK. This demonstrated that CsA@PPTK reduced apoptosis of endothelial cells of heart arteries, and subsequently improved blood supply in infarct area and cardiac function of MI/RI mice.

\section{Discussion}

Currently, there is no ideal treatment method for MI/RI. Many studies suggest that $\mathrm{MI} / \mathrm{RI}$ is actually the result of interaction of multiple factors including cardiomyocytes apoptosis, oxidative stress and inflammatory reaction. Increased ROS leads to apoptosis of cardiomyocytes, inflammatory cell recruitment and inflammatory reaction ${ }^{[38,39]}$. High level of ROS can cause cardiomyocytes apoptosis by affecting mitochondrial membrane potential. ROS can also lead to oxidation of cardiomyocytes membrane and recruitment of inflammatory factors ${ }^{[6]}$. Thus, ROS is an important factor in reperfusion injury. Scavenging ROS has been proved to have protective effect on MI/RI. 
In recent years, ROS-responsive materials have been paid more attention in drug delivery system. This kind of material can not only be used as a carrier, but also can degrade and release drug at sites with high ROS level. Consequently, they display ROS active targeted drug delivery effect. At present, ROS-responsive materials are mainly based on phenylboric acid and thioketal. Thioketal bond can be broken by various ROS such as potassium superoxide $\left(\mathrm{KO}_{2}\right), \mathrm{H}_{2} \mathrm{O}_{2}$, hydroxyl radical $(\cdot \mathrm{OH})$, hypochlorite $\left(\mathrm{ClO}^{-}\right)$and peroxynitrite $\left(\mathrm{ONOO}^{-}\right)$at low concentrations[40]. In this study, based on thioketal, a ROS-responsive material PTK was designed and synthesized. PTK was highly biocompatible and contained a large amount of thioketal bonds. In vitro experiments showed that PTK could eliminate ROS and played a protective role against $\mathrm{H} / \mathrm{R}$ damaged cardiomyocytes through eliminating ROS. PTK could also scavenge ROS in ischemic myocardium in vivo. In addition, PTK released the contained drugs while scavenging ROS, and displayed characteristics of ROS-sensitive drug release. Therefore, PTK was a multifunctional bioactive material with both ROS-responsive drug release and ROS elimination properties.

The cell experiment showed that CsA, CsA@PTK and CsA@PPTK reduced the opening of mPTP and restored the damaged mitochondrial membrane potential of H9c2 cells induced by H/R. Besides, the ROS level in H/R damaged H9c2 cells and its mitochondria was decreased by using @PPTK, CsA@PTK and CsA@PPTK. The cellular uptake experiment indicated that more amount of CsA@PPTK was accumulated in H/R damaged H9c2 cells than CsA and CsA@PTK. Therefore, as compared with @PPTK and CsA@PTK, the scavenging effect of CsA@PPTK on ROS in H/R damaged H9c2 cells and its mitochondria was stronger. The above results demonstrated that CsA@PPTK protected H9c2 cells from H/R injury by strongly blocking the opening of mPTP and eliminating ROS in H/R damaged H9c2 cells.

The key point for targeted drug delivery system to play effective role in MI/RI treatment is that it can target to the area of ischemic myocardium and release drugs rapidly. After being intravenously injected, as compared with CsA@PTK, the amount of CsA@PPTK accumulated in liver of MI/RI mice was significantly reduced. This demonstrated that platelet membrane coated on the surface of nanoparticle markedly reduced the phagocytosis of nanoparticle by MPS. This was because the CD47 on the platelet membrane could inhibit the clearance of CsA@PPTK by MPS [30]. The ex vivo experiment results indicated CsA@PPTK could specifically bind to the endothelial-damaged blood vessel wall. After being intravenously injected to MI/RI mice, CsA@PPTK mainly distributed in apical tissue that was the most severe cardiac ischemia area. This indicated that CsA@PPTK could actively target to ischemic myocardium of MI/RI mice. This was resulted from that transmembrane protein GPIV and GPIX on the platelet membrane could target and bind with the damaged blood vessels, while the integrin-related proteins CD9 and CD81 on the platelet membrane could increase the uptake of CsA@PPTK by cardiomyocytes[41, 42]. The in vitro experimental results showed that CsA@PPTK quickly released CsA at the present of ROS. Thus, CsA@PPTK significantly increased the viability of H/R damaged H9c2 cells. There was a large amount of ROS in ischemic myocardium tissue after reperfusion. Therefore, CsA@PPTK could fast release CsA and reduced the opening of mPTP of cardiomyocytes in cardiac ischemia area. At the same time, CsA@PPTK strongly scavenged ROS in ischemic myocardium tissue. 
Finally, CsA@PPTK alleviated apoptosis of cardiomyocytes and decreased myocardial infarction size in $\mathrm{MI} / \mathrm{RI}$ mice.

Phase one inflammatory reaction induced by M1 type macrophages is closely related to the generation of ROS, which is known as activation of inflammatory macrophages and facilitating recruitment of inflammatory cell to infarct area[43,44]. The phenotype change of macrophages plays a key role in the progress of tissue repair. In the subsequent stages of $\mathrm{Ml} / \mathrm{Rl}$, it is essential for an ideal tissue repair process that pro-inflammatory $\mathrm{M} 1$ type macrophage polarizes to reparative $\mathrm{M} 2$ type macrophage. The increased ratio of M2 type macrophage to M1 type macrophage is of benefits to reducing chronic inflammation and fibrosis in cardiac ischemia tissue. Some studies have also found that timely transformation of M1-type macrophages into M2-type macrophages can effectively increase the repair of infarcted myocardial tissue $[45,46]$. In vivo experimental data indicated that CsA@PPTK obviously increased the ratio of $\mathrm{M} 2$ type macrophage to $\mathrm{M} 1$ type macrophage in heart tissue. At the same time, in vivo experimental results also showed that in CsA@PPTK treated group, the number of M1 type macrophages and M2 type macrophages markedly decreased, indicating the number of macrophages recruited to ischemic myocardial tissue decreased. This was resulted from the decrease in heart injury of MI/RI mice by CsA@PPTK, which further resulted in the decrease in the number of recruited macrophages. Besides, CsA@PPTK significantly reduced myocardial fibrosis area as compared with CsA, @PPTK and CsA@PTK. CsA@PPTK greatly decreased the systemic inflammatory reaction by reducing IL$1 \beta$ and TGF- $\beta$ concentration in serum of MI/RI mice. This was resulted from following three reasons. Firstly, CsA@PPTK improved the oxidized microenvironment of MI/RI by scavenging ROS in cardiac ischemia area. Secondly, CsA@PPTK reduced apoptosis of cardiomyocytes in ischemic myocardium tissue by releasing CsA to inhibit the opening of MPTP. The vicious cycle between the burst release of ROS and apoptosis of cardiomyocytes in ischemic myocardium tissue was blocked. Finally, CsA can inhibit the immune response mediated by $T$ lymphocytes, and reduce the release of various lymphocytes such as IL-2, IL-3, IFN- $y$ and the expression of IL-2R. Consequently, recruitment of inflammatory cells to damaged myocardium tissue was weakened and inflammatory reaction was alleviated.

IL-1 $\beta$ is a pro-inflammatory factor. Our study showed that MI/RI significantly increased the level of IL-1 $\beta$ in serum, while CsA, @PPTK, CsA@PTK and CsA@PPTK reduced the level of IL-1 $\beta$ to a certain extent. CsA@PPTK displayed the most significant effect on the reduction of IL-1 $1 \beta$. These results indicated that CsA@PPTK displayed anti-inflammatory effect, which was consistent with the results of immunofluorescence staining. TGF- $\beta$, secreted by anti-inflammatory macrophages, has been shown to play a protective role in the myocardial matrix by inhibiting the synthesis of metalloproteinases and inducing the synthesis of protease inhibitors $[47,48]$. However, TGF- $\beta$ overexpression can also induce the transformation of cardiac fibroblasts into myofibroblasts, leading to myocardial fibrosis and cardiac remodeling ${ }^{[49]}$. Our study showed that TGF- $\beta$ level increased to a certain extent after MI/RI, which resulted from compensation after myocardial injury. There was no significant change in the level of TGF- $\beta$ in the CsA@PPTK group compared with the sham group, which suggested that CsA@PPTK could better maintain the homeostasis of injured myocardial tissue, and subsequently reduced cardiac remodeling. 
The results showed that the number of Tregs in the myocardial infarction area increased significantly 3 days after reperfusion, which was due to the fact that circulating Tregs infiltrated into the myocardial tissue through vascular wall in the early stage of myocardial injury to antagonize excessive inflammatory response. CsA displayed an inhibitory effect on Tregs. Thus, a significant decrease in Tregs in cardiac tissue was observed after free CsA was administered. Studies have found that ROS scavenger can increase the number of Tregs. Therefore, the increase of Tregs by CsA@PPTK might be due to the ROS scavenging effect of PTK, which counteracted the inhibitory effect of CsA on Tregs, and this counteraction effect was much stronger at higher doses.

It was reported that Tregs increased in myocardial tissue after reperfusion in mice. Tregs alleviated heart damage of MI/RI mice by inhibiting apoptosis of cardiomyocytes and neutrophil infiltration and reducing infarct size[11, 12]. Besides, Tregs inhibited the activity of cytotoxic T cells and pro-inflammatory M1 type macrophage, and then suppressed local inflammatory response and systemic cytotoxic response induced by myocardial infarction, thereby reducing ventricular remodeling and improving cardiac function[50, 51]. The experiment results indicated that CsA@PPTK augmented Tregs in ischemic myocardium tissue. In a word, by reprogram Tregs generation, the ratio of M2 type macrophage to M1 type macrophage and redox microenvironment, CsA@PPTK alleviated inflammatory reaction and subsequently reduced myocardial fibrosis and the remodeling of left ventricle. In general, CsA@PPTK could not only simulate the effect of Tregs through anti-apoptotic, anti-inflammatory and anti-oxidant effects, but also played a synergistic protective role on MI/RI by directly increasing the number of Tregs in myocardial infarction area.

MMP-9 increased sharply after myocardial infarction, and it was closely related to the remodeling of left ventricle. Elevated MMP-9 levels have been found in ventricular remodeling tissue after AMI[52, 53]. In $\mathrm{MI} / \mathrm{RI}$ animal models, the expression of MMP-9 was increased with the prolongation of heart injury. The activity of MMP-9 is positively correlated with the severity of AMI, and the mortality of Ml/RI animals was significantly increased with the increase of MMP-9 expression. In the process of infarction, inflammatory reaction plays an important role in expression of MMP-9. CsA@PPTK significantly alleviated inflammatory reaction in MI/RI mice, which resulted in the decrease of MMP-9 expression in left ventricle[54]. Furthermore, the experimental data also demonstrated that CsA@PPTK markedly attenuated left ventricular remodeling by reducing MMP-9 expression in left ventricle anterior wall of MI/RI mice.

The CX43 is the most important protein in the gap junction of the left ventricular muscle[37]. The cardiac electrochemical impulse is mainly transmitted to the cardiomyocytes of the left ventricle through CX43 to maintain the rhythmic contraction of the left ventricle. Under normal circumstances, CX43 mainly exists as a phosphorylated status. When myocardial ischemia occurs, the expression of CX43 will be reduced, and CX43 protein is dephosphorylated, which eventually induces arrhythmias. The experimental results showed that CsA@PPTK significantly increased the expression of CX43 as compared with free CsA and CsA@PTK, indicating CsA@PPTK greatly increased the activity of cardiomyocytes and cardiac function in the late repair period of $\mathrm{MI} / \mathrm{RI}$.

\section{Conclusion}


Platelet membrane-based Tregs biomimetic nanoparticle (CsA@PPTK) is suitable for ischemia myocardium targeting drug delivery and ROS-triggered drug release. CsA@PPTK significantly reduced the remodeling of the left ventricle and enhanced heart protective effect through reprograming inflammatory and redox microenvironment and attenuating apoptosis of cardiomyocytes at infarct tissue.

\section{Declarations}

\section{Ethics approval and consent to participate}

All the animals received care in terms of the guidelines for the Care and Use of Laboratory Animals and the animal experiments were approved by the Air Force Medical University Institutional Animal Care and Utilization Committee (No: IACUC-20190505).

\section{Consent for publication}

Not applicable.

\section{Availability of data and materials}

All data generated or analyzed during this study are included in this published article and its supplementary information file.

\section{Competing interests}

There is no competing interest.

\section{Funding}

This research was supported by the National Nature Science Foundation (No. 82003662) and Nature Science Foundation of Shaanxi Province (No. 2020JM-331). Shaanxi Province Key Research and Development Projects of China (2021ZDLSF03-08)

\section{Authors' contributions}

$\mathrm{FL}, \mathrm{YC}$, and $\mathrm{DL}$ performed the experiments and analysis. $\mathrm{ML}$ and QJ performed investigation and visualization. BZ and QM performed conceptualization and methodology. SZ performed project administration and funding acquisition. All authors read and approved the final manuscript.

\section{Acknowledgements}

Not applicable

\section{References}


1. Su T, Huang K, Ma H, Liang H, Dinh PU, Chen J, et al. Platelet-Inspired Nanocells for Targeted Heart Repair After Ischemia/Reperfusion Injury. Adv Funct Mater. 2019; 29.

2. Vicencio JM, Yellon DM, Sivaraman V, Das D, Boi-Doku C, Arjun S, et al. Plasma exosomes protect the myocardium from ischemia-reperfusion injury. J Am Coll Cardiol. 2015; 65:1525.

3. Chouchani ET, Pell VR, Gaude E, Aksentijevic D, Sundier SY, Robb EL, et al. Ischaemic accumulation of succinate controls reperfusion injury through mitochondrial ROS. Nature. 2014; 515:431.

4. Lloyd-Jones D, Adams RJ, Brown TM, Carnethon M, Dai S, De Simone G, et al. Executive summary: heart disease and stroke statistics-2010 update: a report from the American Heart Association. Circulation. 2010; 121:948.

5. Velagaleti RS, Pencina MJ, Murabito JM, Wang TJ, Parikh NI, D'Agostino RB, et al. Long-term trends in the incidence of heart failure after myocardial infarction. Circulation. 2008; 118:2057.

6. Hausenloy DJ, Yellon DM. Myocardial ischemia-reperfusion injury: a neglected therapeutic target. J Clin Invest. 2013; 123:92.

7. Weil BR, Neelamegham S. Selectins and Immune Cells in Acute Myocardial Infarction and Postinfarction Ventricular Remodeling: Pathophysiology and Novel Treatments. Front Immunol. 2019; 10:300.

8. Rurik JG, Aghajanian H, Epstein JA. Immune Cells and Immunotherapy for Cardiac Injury and Repair. Circ Res. 2021; 128:1766.

9. Weirather J, Hofmann UD, Beyersdorf N, Ramos GC, Vogel B, Frey A, et al. Foxp3+ CD4+ T cells improve healing after myocardial infarction by modulating monocyte/macrophage differentiation. Circ Res. 2014; 115:55.

10. Sharir R, Semo J, Shimoni S, Ben-Mordechai T, Landa-Rouben N, Maysel-Auslender S, et al. Experimental myocardial infarction induces altered regulatory $T$ cell hemostasis, and adoptive transfer attenuates subsequent remodeling. PLoS One. 2014; 9:e113653.

11. Tang TT, Yuan J, Zhu ZF, Zhang WC, Xiao H, Xia N, et al. Regulatory T cells ameliorate cardiac remodeling after myocardial infarction. Basic Res Cardiol. 2012; 107:232.

12. Xia N, Lu Y, Gu M, Li N, Liu M, Jiao J, et al. A Unique Population of Regulatory T Cells in Heart Potentiates Cardiac Protection From Myocardial Infarction. Circulation. 2020; 142:1956.

13. Wang H, Hou L, Kwak D, Fassett J, Xu X, Chen A, et al. Increasing Regulatory T Cells With Interleukin-2 and Interleukin-2 Antibody Complexes Attenuates Lung Inflammation and Heart Failure Progression. Hypertension. 2016; 68:114.

14. Nighoghossian N, Ovize M, Mewton N, Ong E, Cho TH. Cyclosporine A, a Potential Therapy of Ischemic Reperfusion Injury. A Common History for Heart and Brain. Cerebrovascular Diseases. 2016; 42:309.

15. el Tayar N, Mark AE, Vallat P, Brunne RM, Testa B, van Gunsteren WF. Solvent-dependent conformation and hydrogen-bonding capacity of cyclosporin $A$ : evidence from partition coefficients and molecular dynamics simulations. J Med Chem. 1993; 36:3757. 
16. Zhang CX, Cheng Y, Liu DZ, Liu M, Cui H, Zhang BL, et al. Mitochondria-targeted cyclosporin A delivery system to treat myocardial ischemia reperfusion injury of rats. J Nanobiotechnology. 2019; 17:18.

17. Liu Y, Shi J. Antioxidative nanomaterials and biomedical applications. Nano Today. 2019; 27:146.

18. Cheng Y, Liu D-Z, Zhang C-x, Cui H, Liu M, Zhang B-I, et al. Mitochondria-targeted antioxidant delivery for precise treatment of myocardial ischemia-reperfusion injury through a multistage continuous targeted strategy. Nanomedicine: Nanotechnology, Biology and Medicine. 2019; 16:236.

19. Yao Y, Ding J, Wang Z, Zhang H, Xie J, Wang Y, et al. ROS-responsive polyurethane fibrous patches loaded with methylprednisolone (MP) for restoring structures and functions of infarcted myocardium in vivo. Biomaterials. 2020; 232:119726.

20. Hoang TT, Smith TP, Raines RT. A Boronic Acid Conjugate of Angiogenin that Shows ROS-Responsive Neuroprotective Activity. Angewandte Chemie International Edition. 2017; 56:2619.

21. Gustafson HH, Holt-Casper D, Grainger DW, Ghandehari H. Nanoparticle Uptake: The Phagocyte Problem. Nano Today. 2015; 10:487.

22. Cao X, Hu Y, Luo S, Wang Y, Gong T, Sun X, et al. Neutrophil-mimicking therapeutic nanoparticles for targeted chemotherapy of pancreatic carcinoma. Acta Pharm Sin B. 2019; 9:575.

23. Boada C, Zinger A, Tsao C, Zhao P, Martinez JO, Hartman K, et al. Rapamycin-Loaded Biomimetic Nanoparticles Reverse Vascular Inflammation. Circ Res. 2020; 126:25.

24. Lin Y, Liu J, Bai R, Shi J, Zhu X, Liu J, et al. Mitochondria-Inspired Nanoparticles with Microenvironment-Adapting Capacities for On-Demand Drug Delivery after Ischemic Injury. ACS Nano. 2020; 14:11846.

25. Coenen DM, Mastenbroek TG, Cosemans J. Platelet interaction with activated endothelium: mechanistic insights from microfluidics. Blood. 2017; 130:2819.

26. Schanze N, Bode C, Duerschmied D. Platelet Contributions to Myocardial Ischemia/Reperfusion Injury. Front Immunol. 2019; 10:1260.

27. Tang J, Su T, Huang K, Dinh PU, Wang Z, Vandergriff A, et al. Targeted repair of heart injury by stem cells fused with platelet nanovesicles. Nat Biomed Eng. 2018; 2:17.

28. $\otimes \mathrm{Li} \mathrm{Z}$, Hu S, Cheng K. Platelets and their biomimetics for regenerative medicine and cancer therapies. J Mater Chem B. 2018; 6:7354.

29. Hu Q, Sun W, Qian C, Wang C, Bomba HN, Gu Z. Anticancer Platelet-Mimicking Nanovehicles. Adv Mater. 2015; 27:7043.

30. Hu CM, Fang RH, Wang KC, Luk BT, Thamphiwatana S, Dehaini D, et al. Nanoparticle biointerfacing by platelet membrane cloaking. Nature. 2015; 526:118.

31. Shim MS, Xia Y. A reactive oxygen species (ROS)-responsive polymer for safe, efficient, and targeted gene delivery in cancer cells. Angew Chem Int Ed Engl. 2013; 52:6926.

32. Wei X, Gao J, Fang RH, Luk BT, Kroll AV, Dehaini D, et al. Nanoparticles camouflaged in platelet membrane coating as an antibody decoy for the treatment of immune thrombocytopenia. 
Biomaterials. 2016; 111:116.

33. Qiao S, Cheng Y, Liu M, Ji Q, Zhang B, Mei Q, et al. Chemoattractants driven and microglia based biomimetic nanoparticle treating TMZ-resistant glioblastoma multiforme. Journal of Controlled Release. 2021; 336:54.

34. Wang W, Chen J, Li M, Jia H, Han X, Zhang J, et al. Rebuilding Postinfarcted Cardiac Functions by Injecting TIIA@PDA Nanoparticle-Cross-linked ROS-Sensitive Hydrogels. ACS Appl Mater Interfaces. 2019; 11:2880.

35. Kam N, Jessop TC, Wender PA, Dai H. Nanotube Molecular Transporters: Internalization of Carbon Nanotube -- Protein Conjugates into Mammalian Cells. Journal of the American Chemical Society. 2004; $126: 6850$.

36. Ruparelia N, Chai JT, Fisher EA, Choudhury RP. Inflammatory processes in cardiovascular disease: a route to targeted therapies. Nat Rev Cardiol. 2017; 14:133.

37. Jongsma HJ, Wilders R, . Gap junctions in cardiovascular disease. circulation research. 2000; 86:1193.

38. Li J, Shu Y, Hao T, Wang Y, Qian Y, Duan C, et al. A chitosan-glutathione based injectable hydrogel for suppression of oxidative stress damage in cardiomyocytes. Biomaterials. 2013; 34:9071.

39. Zhu Y, Matsumura Y, Velayutham M, Foley LM, Hitchens TK, Wagner WR. Reactive oxygen species scavenging with a biodegradable, thermally responsive hydrogel compatible with soft tissue injection. Biomaterials. 2018; 177:98.

40. Zhang W, Hu X, Shen Q, Xing D. Mitochondria-specific drug release and reactive oxygen species burst induced by polyprodrug nanoreactors can enhance chemotherapy. Nature Communications. 2019; 10.

41. Nieswandt B, Watson SP. Platelet-collagen interaction: is GPVI the central receptor? Blood. 2003; 102:449.

42. Zhang F, Kotha J, Jennings LK, Zhang XA. Tetraspanins and vascular functions. Cardiovasc Res. 2009; 83:7.

43. Jeong HY, Kang WS, Hong MH, Jeong HC, Shin MG, Jeong MH, et al. 5-Azacytidine modulates interferon regulatory factor 1 in macrophages to exert a cardioprotective effect. Sci Rep. 2015; 5:15768.

44. Kim YS, Jeong HY, Kim AR, Kim WH, Cho H, Um J, et al. Natural product derivative BIO promotes recovery after myocardial infarction via unique modulation of the cardiac microenvironment. Sci Rep. 2016; 6:30726.

45. Han J, Kim YS, Lim M-Y, Kim HY, Kong S, Kang M, et al. Dual Roles of Graphene Oxide To Attenuate Inflammation and Elicit Timely Polarization of Macrophage Phenotypes for Cardiac Repair. ACS Nano. 2018; 12:1959.

46. Lüscher T. The sooner, the better: anti-inflammation in acute myocardial infarction. European Heart Journal. 2020; 41:4100. 
47. Bejerano T, Etzion S, Elyagon S, Etzion Y, Cohen S. Nanoparticle Delivery of miRNA-21 Mimic to Cardiac Macrophages Improves Myocardial Remodeling after Myocardial Infarction. Nano Lett. 2018; 18:5885.

48. Schiller M, Javelaud D, Mauviel A. TGF- $\beta$-induced SMAD signaling and gene regulation: consequences for extracellular matrix remodeling and wound healing. Journal of Dermatological Science. 2004; 35:83.

49. Leask A, Abraham DJ. TGF- $\beta$ signaling and the fibrotic response. Faseb Journal Official Publication of the Federation of American Societies for Experimental Biology. 2004; 18:816.

50. Saxena A, Do Ba Czewski M, Rai V, Haque Z, Frangogiannis NG. Regulatory T cells are recruited in the infarcted mouse myocardium and may modulate fibroblast phenotype and function. Am J Physiol Heart Circ Physiol. 2014; 307:H1233.

51. Van D, Nahrendorf M, Piek J. Healing and adverse remodelling after acute myocardial infarction: role of the cellular immune response. Heart. 2012; 98:1384.

52. King MK, Coker ML, Goldberg A, McElmurray JH, 3rd, Gunasinghe HR, Mukherjee R, et al. Selective matrix metalloproteinase inhibition with developing heart failure: effects on left ventricular function and structure. Circ Res. 2003; 92:177.

53. Mukherjee R, Brinsa TA, Dowdy KB, Scott AA, Baskin JM, Deschamps AM, et al. Myocardial infarct expansion and matrix metalloproteinase inhibition. Circulation. 2003; 107:618.

54. Halade GV, Jin YF, Lindsey ML. Matrix metalloproteinase (MMP)-9: a proximal biomarker for cardiac remodeling and a distal biomarker for inflammation. Pharmacol Ther. 2013; 139:32.

\section{Supplementary}

Schema 1 is available in supplementary section

\section{Figures}

\section{Figure 1}

Characterizations of the CsA@PPTK. (A) TEM image. (B) Particle size distribution and zeta potential. (C) Confocal fluorescent microscopy images of CsA@PPTK. (red: platelet membrane, green: CsA@PTK labeled with Coumarin 6). (D) Protein content visualization of platelet, platelet membrane, CsA@PPTK and CsA@PTK run on SDS-PAGE. (E) Stability of CsA@PPTK in PBS solution.

\section{Figure 2}


In vitro ROS responsibility of CsA@PPTK. (A) The appearance changes of CsA@PPTK NPs in PBS and NaClO-containing PBS (pH7.4) solution. (B) Cumulative drug release of CsA@PPTK in PBS (pH7.4) and NaClO-containing PBS solution. (C) The change of solution color after CsA@PPTK was added to DPPH solution. (D) Statistical results of absorbance values of DPPH solution at $520 \mathrm{~nm} . n=3, x \pm$ SD; ${ }^{\star * \star} p<0.001$. (E) The effect of CsA@PPTK viability of H/R injured H9C2 cells. $n=6, x \pm S D$; ${ }^{* \star} p<0.01$.

\section{Figure 3}

The protective effects of CsA@PPTK on H/R injured H9C2 cells. (A) Schematic diagram of the establishment of $\mathrm{H} / \mathrm{R}$ injured $\mathrm{H} 9 \mathrm{C} 2$ cell model. (B) Representative image of MitoSOX fluorescence intensity measured in H/R injury H9c2 cells by CLSM (20x). (C) Statistical results of MitoSOX fluorescence intensity. (D) Calcien AM fluorescence intensity measured in H/R injury H9c2 cells by flow cytometry. (E) Statistical results of Calcien AM fluorescence intensity. (F) Statistical results of the ratio of $\mathrm{red} /$ green fluorescence intensity in $\mathrm{H} / \mathrm{R}$ injury $\mathrm{H} 9 \mathrm{C} 2$ cells after JC-1 staining. $\mathrm{n}=3, \mathrm{x} \pm \mathrm{SD} ; * \mathrm{p}<0.05$,

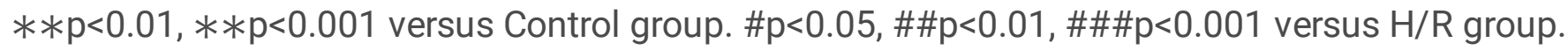
$\# \# \# p<0.001$ versus CsA group.

\section{Figure 4}

Targeted distribution of CsA@PPTK in ex vivo and in vivo. (A) Representative image of Coumarin fluorescence intensity in denude aorta. (B) Statistical results of Coumarin fluorescence intensity. (C)

Distribution of Cy7.5@PPTK in the hearts of sham and MI/RI mice. (D) The distribution of Cy7.5@PPTK in the heart slice of sham and MI/RI mice.

\section{Figure 5}

Left ventricular function of MI/RI mice at 28 days after administration of CsA@PPTK. (A) Representative echocardiography of mice. (B) Statistical results of LVEF. (C) Statistical results of FS in different groups.

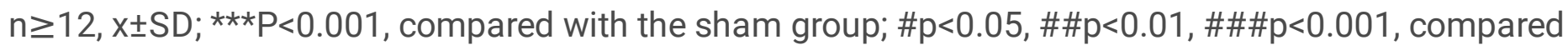

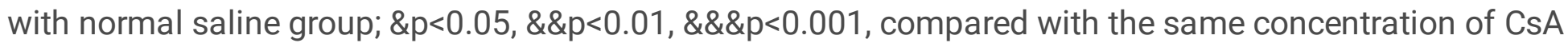
group; $\$ \mathrm{p}<0.01$, compared with CsA@PTK group

\section{Figure 6}

Effect of CsA@PPTK on immune microenvironment of myocardial tissue. (A) Representative images of CD86 staining (green) and CD206 staining (red) in myocardial tissue of MI/RI mice at day 4 after reperfusion. (B) The statistical results of fluorescence intensity of CD86/CD206. (C) IL-1 $\beta$ concentration in serum of MI/RI mice at day 4 after reperfusion. (D) TGF- $\beta$ concentration in serum of MI/RI mice at day 
4 after reperfusion. (E) Representative images of Tregs staining (green) in myocardial tissue of MI/RI mice at day 4 after reperfusion. (F) The statistical results of fluorescence intensity of Foxp3. $n=3, x \pm S D$; $\star \star P<0.01,{ }^{* \star *} p<0.001$ compared with sham group; $\# p<0.05$, \#\#p<0.01, compared with normal saline group.

\section{Figure 7}

The protective effect of CsA@PPTK on MI/RI mice. (A) Representative images of TUNEL (red) staining in myocardial tissue of MI/RI mice at day 7 after reperfusion. (B) The percentage of apoptic cells. (C) Representative image of Masson and H\&E staining of myocardial tissue of MI/RI mice at 28 days after reperfusion. (D) Statistical results of the scar area. (E) Statistical results of the LV area. $n \geq 5, \bar{x} \pm S D$; ${ }^{\star *} \mathrm{P}<0.01,{ }^{* \star *} \mathrm{p}<0.001$ compared with sham group; $\# \mathrm{p}<0.05, \# \# \mathrm{p}<0.01, \# \# \# \mathrm{p}<0.001$ compared with normal saline group; $\& p<0.05, \& \& p<0.01$, compared with the same concentration of CsA group.

\section{Figure 8}

The effect of CsA@PPTK on expression of CX43, MMP-9 and a-SMA in myocardial tissue. (A) Representative images of CX43 staining (red) in myocardial tissue of MI/RI mice at 28 days after reperfusion. (B) The statistical results of CX43 staining. (C) Representative images of MMP-9 staining (green) and a-SMA staining (red) in myocardial tissue of MI/RI mice at 28 days after reperfusion. (D) The statistical results of MMP-9 staining. (E) The statistical results of a-SMA staining. ${ }^{\star *} P<0.01,{ }^{\star \star} \mathrm{p}<0.001$

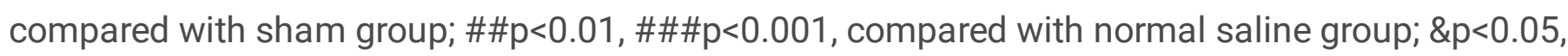
$\& \& p<0.01$, compared with the same concentration of CsA group.

\section{Supplementary Files}

This is a list of supplementary files associated with this preprint. Click to download.

- scheme1.tif

- SupportingInformation.docx 